Pacific Journal of Mathematics

A REMARK ON THE NIJENHUIS TENSOR

March 1962 


\title{
A REMARK ON THE NIJENHUIS TENSOR
}

\author{
EDWARD T. KOBAYASHI
}

The vanishing of the Nijenhuis tensor of the almost complex structure is known to give the integrability of the almost complex structure $[3,7]$. In order to generalize this fact, we consider a vector 1 -form $h$ on a manifold $M[4]$, whose Jordan canonical form at all points on $M$ is equal to a fixed matrix $\mu$. Following the idea of E. Cartan, we say that such a vector 1 -form is 0 -deformable [2]. The frames $z$ at $x$ such that $z^{-1} h_{x} z=$ $\mu$ define a subbundle of the frame bundle over $M$, as $x$ runs through $M$, and the subbundle is called a $G$-structure defined by $h$ [1]. We find that for a certain type of 0 -deformable $h$, the vanishing of the Nijenhuis tensor of $h$ is sufficient for the $G$-structure to be integrable (Theorem, $\S 2)$. In $\S 5$ we give an example of a 0 -deformable derogatory nilpotent vector 1 -form, whose Nijenhuis tensor vanishes, but whose $G$-structure is not integrable.

1. Vector forms and distributions. As usual, we begin by stating, that all the objects we encounter in this paper are assumed to be $C^{\infty}$.

Let $M$ be a manifold, $T_{x}$ the tangent space at point $x$ of $M, T$ the tangent bundle over $M, T^{(p)}$ the vector bundle of tangential covariant $p$-vectors of $M$. A vector $p$-form is a cross-section of $T \otimes T^{(p)}$. The collection of all vector $p$-forms over $M$ is denoted by $\Psi_{p}$. We notice that a vector 1 -form is nothing but a law that assigns a linear transformation to each tangent space $T_{x}$ at point $x$ of $M$.

We list some definitions and lemmas of the theory of vector forms [4], which we use in the sequel.

If $P \in \Psi_{p}, Q \in \Psi_{q}$, then $P \pi Q \in \Psi_{p+q-1}$ is defined by

$$
\begin{aligned}
& (p \pi Q)\left(u_{1}, \cdots, u_{p+q-1}\right) \\
= & \frac{1}{(p-1) ! q !} \sum_{\alpha}|\alpha| P\left(Q\left(u_{\alpha_{1}} \ldots, u_{\alpha_{p}}\right), u_{\alpha_{p+1}} \ldots, u_{\alpha_{p+q-1}}\right)
\end{aligned}
$$

where $\alpha$ runs through all the permutations of $(1,2, \cdots, p+q-1)$, and $|\alpha|$ denotes the signature of the permutation $\alpha$.

If $h$ is a vector 1 -form and $P$ is a vector $p$-form, we write $h P$ instead of $h \pi p$. In particular if $p=h$, we write $h \pi h$ as $h^{2}$. In general, $h \pi \underset{k \text { times }}{h} \cdots \pi h$ is written as $h^{k}$, and this agrees with the usual notation,

Received November 13, 1961. This research was supported in part by National Science Foundation grant G 14736. The author wishes to thank Professor H. C. Wang for first suggesting this problem and for the subsequent discussion we had on this paper. The author is also obliged to the referee whose comments helped to correct and clarify some arguments in this paper. 
when we consider $h$ as a linear transformation of the tangent space at each point of the manifold $M$.

Let $h$ and $k$ be two vector 1 -forms. The bracket $[h, k]$ of $h$ and $k$ is a vector 2 -form defined by

$$
\begin{aligned}
{[h, k](u, v)=} & {[h u, k v]+[k u, h v]-k[h u, v]-h[k u, v] } \\
& -k[u, h v]-h[u, k v]+k h[u, v]+h k[u, v],
\end{aligned}
$$

where $u$ and $v$ are vector fields over $M$. If $h=k$, we obtain the tensor $[h, h]$, generally known as the Nijenhuis tensor:

$$
\frac{1}{2}[h, h](u, v)=[h u, h v]-h[h u, v]-h[u, h v]+h^{2}[u, v] .
$$

If $h, k$ and $l$ are vector 1 -forms, using (2), we can obtain

$$
[h l, k]+[h, k l]-[h, k] \pi l=h[l, k]+k[l, h]
$$

(cf. (6.7) [4]).

LEMMA 1.1. Let $h$ be a vector 1-form, then

$$
\left[h^{k}, h^{l}\right]=\frac{1}{2} \sum_{\substack{a+b+c+k=k+l-2 \\ 0 \leqq b \leq l-1 \\ 0 \leqq c \leqq l}} h^{a}\left\{\left([h, h] \pi h^{b}\right) \pi h^{c}-[h, h] \pi h^{b+c}\right\} .
$$

Proof. By replacing $h, k$ and $l$ by $h, h$ and $h^{k}$ in (4), we obtain

$$
\left[h^{k}, h\right]=h\left[h^{k-1}, h\right]+\frac{1}{2}[h, h] \pi h^{k-1},
$$

which gives us

$$
\left[h^{k}, h\right]=\frac{1}{2} \sum_{i=1}^{k} h^{i-1}[h, h] \pi h^{k-i} .
$$

Again, replacing $h, k$ and $l$ in (4) by $h^{k}, h$ and $h^{l-1}$, we obtain

$$
\left[h^{k+l-1}, h\right]+\left[h^{k}, h^{\imath}\right]-\left[h^{k}, h\right] \pi h^{l-1}=h^{k}\left[h^{l-1}, h\right]+h\left[h^{l-1}, h^{k}\right] .
$$

Using (7) and (8) yields

$$
\begin{aligned}
& {\left[h^{k}, h^{l}\right]=h\left[h^{k}, h^{l-1}\right]} \\
& \quad+\frac{1}{2} \sum_{i=1}^{k} h^{i-1}\left\{\left([h, h] \pi h^{k-i}\right) \pi h^{l-1}-[h, h] \pi h^{k-i+l-1}\right\},
\end{aligned}
$$

and repeating the reduction we obtain (5).

LEMMA 1.2. Let $h$ be a vector 1-form on $M$, whose rank is constant 
in a neighbourhood of each point $x$ of $M$. If $[h, h]=0$, the distribution $x \rightarrow h_{x} T_{x}$ is completely integrable.

Proof. By Frobenius' theorem we have to show that the bracket of any two vector fields of the form $h u, h v$ belongs to the distribution. This follows from $[h, h]=0$ and (3):

$$
[h u, h v]=h[h u, v]+h[u, h v]-h^{2}[u, v] .
$$

We recall that a necessary and sufficient condition for a distribution to be completely integrable can be given as follows:

Let $\theta$ be an $r$-dimensional distribution $x \rightarrow \theta(x)$ on an $m$-dimensional manifold $M$. For each $x_{0} \in M$, let $U$ be a neighbourhood of $x_{0}$ and $L_{1}, \cdots, L_{r}$ be vector fields on $U$ such that $\left(L_{1}\right)_{x}, \cdots,\left(L_{r}\right)_{x}$ span $\theta(x)$ for each $x \in U$. Then $\theta$ is completely integrable if and only if for each $x_{0} \in M$, there exist $m-r$ independent functions $\psi^{1}, \cdots, \psi^{m-r}$ defined on a neighbourhood $V \subset U$ of $x_{0}$ such that

$$
L_{i} \psi^{j}=0 \text {, for } 1 \leqq i \leqq r, 1 \leqq j \leqq m-r \text { on } V \text {. }
$$

Using this it is easy to prove,

LEMMA 1.3. If $\theta_{1}, \cdots, \theta_{g}$ are completely integrable distributions of dimensions $r_{1}, \cdots, r_{g}$ on $M$, such that

$$
\theta_{1}(x)+\theta_{2}(x)+\cdots+\theta_{g}(x)=T_{x}(\text { direct sum })
$$

for each $x \in M$, then for each point $x_{0} \in M$, there exists a coordinate neighbourhood $U$ of $x_{0}$ with coordinate functions $x^{1}, \cdots, x^{m}$ such that for each $j$

$$
x^{1}=\xi^{1}, \cdots, x^{r_{1}+\cdots+r_{j-1}}=\xi^{r_{1}+\cdots+r_{j-1}}, x^{r_{1}+\cdots+r_{j}+1}=\xi^{r_{1}+\cdots+r_{j}+1}, \cdots, x^{m}=\xi^{m}
$$

gives an integral manifold of $\theta_{j}$ contained in $U$.

2. The integrability of a 0 -deformable vector 1 -form. Let $h$ be a vector 1-form, defined on $M$, whose characteristic polynomial has constant coefficients on $M$. Let the decomposition of the characteristic polynomial be

$$
\left\{p_{1}(\lambda)\right\}^{a_{1}}\left\{p_{2}(\lambda)\right\}^{a_{2}} \cdots\left\{p_{g}(\lambda)\right\}^{a_{g}}
$$

where $p_{i}(\lambda), i=1, \cdots, g$ are polynomials in $\lambda$, irreducible over the reals, and $\left(p_{i}(\lambda), p_{j}(\lambda)\right)=1$, if $i \neq j$. It is easy to verify [5, pp 130-132], that we can get polynomials $e_{1}(\lambda), e_{2}(\lambda), \cdots, e_{g}(\lambda)$ in $\lambda$, with constant coefficients, such that $\sum_{i=1}^{g} e_{i}(h)=I,\left\{e_{i}(h)\right\}^{2}=e_{i}(h), e_{i}(h) \cdot e_{j}(h)=0$ for $i \neq j$, and 


$$
e_{i}\left(h_{x}\right) T_{x}=\left\{u_{x} \in T_{x} \mid\left\{p_{i}\left(h_{x}\right)\right\}^{a_{i}} u_{x}=0\right\} .
$$

Let $\theta_{i}$ denote the distribution $x \rightarrow e_{i}\left(h_{x}\right) T_{x}$. If we assume $[h, h]=0$, then by Lemma 1.1, because $e_{i}(h)$ is a polynomial in $h$ with constant coefficients, we see that $\left[e_{i}(h), e_{i}(h)\right]=0$. Hence, by Lemma 1.2, $\theta_{i}$ is completely integrable.

Definition. A vector 1 -form $h$ on $M$ is said to be 0 -deformable, if for all $x \in M$, the Jordan canonical form of $h_{x}$ is equal to a fixed matrix $\mu[2]$.

Note that a 0 -deformable vector 1 -form has a characteristic polynomial with constant coefficients.

A frame at $x \in M$ is an isomorphism $z$ from $R^{m}$ onto $T_{x}$, where $m$ is the dimension of $M$. For a 0 -deformable vector 1 -form $h$, the frames $z$ at $x$ such that $z^{-1} h_{x} z=\mu$ define a subbundle $H$ of the frame bundle over $M$, as $x$ runs through $M . \quad H$ is called the $G$-structure defined by $h[1]$.

Definition. A $G$-structure $H$ defined by $h$ is said to be integrable, if for each point $x$ of $M$ there exists a coordinate neighbourhood $U$ of $x$ with a coordinate system $\left\{x^{1}, \cdots, x^{m}\right\}$ such that the frame $\left\{\left(\partial / \partial x^{1}\right)_{x^{\prime}}, \cdots\right.$, $\left.\left(\partial / \partial x^{m}\right)_{x^{\prime}}\right\}$ belongs to the subbundle $H$ for all $x^{\prime} \in U$. We shall say that these coordinate functions are associated with the integrable $G$-structure $H$.

Clearly, $H$ is integrable if and only if, for each point $x$ of $M$, we can find a local coordinate system around $x$, in which the coordinate expression of $h$ is $\mu$.

We are interested in finding a sufficient condition for a $G$-structure defined by a 0 -deformable vector 1 -form $h$ to be integrable. We now assume $[h, h]=0$. By the argument above we know that the distributions $\theta_{i}$ associated to the irreducible factors $p_{i}(\lambda)$ are all completely integrable, so by Lemma 1.3, for each point $x_{0}$ of $M$ there is a coordinate system $\left\{x^{1}, \cdots, x^{m}\right\}$ on a neighbourhood $U$ of $x_{0}$, and the integral manifolds of $\theta_{i}$ contained in $U$ are given by coordinate slices.

In $U$ take a point given by coordinates $\left(\xi^{1}, \cdots, \xi^{m}\right)$. For each $i$, let $x^{1}=\xi^{1}, \cdots, x^{r_{i-1}}=\xi^{r_{i-1}}, x^{r_{i}+1}=\xi^{r_{i}+1}, \cdots, x^{m}=\xi^{m}$ give an integral manifold $M_{i}$ of $\theta_{i}$ in $U$, where $r_{i}=m_{1}+m_{2}+\cdots+m_{i}$ and $m_{i}=$ dimension of $\theta_{i}$. Consider the restriction $h_{i}$ of $h$ on $M_{i}$. Notice that we can view $h_{i}$ as a vector 1-form on an open set of $M_{i}$, depending on $m-m_{i}$ parameters $x^{1}, \cdots, x^{r_{i-1}}, x^{r_{i}+1}, \cdots, x^{m}$ in such the way that $h_{i}$ is $C^{\infty}$ with respect to the coordinates on $M_{i}$ and the parameters together. The characteristic polynomial of $h_{i}$ is $\left\{p_{i}(\lambda)\right\}^{a_{i}}$ and the minimum polynomial of $h_{i}$ is $\left\{p_{i}(\lambda)\right\}^{v_{i}}$, where $\prod_{i=1}^{g}\left\{p_{i}(\lambda)\right\}^{v_{i}}$ is the minimum polynomial of $h$; $h_{i}$ is a 0 -deformable vector 1 -form on $M_{i}$, and $\left[h_{i}, h_{i}\right]=0$. If for each $i$, 
the $G_{i}$-structure defined by $h_{i}$ on $M_{i}$ is integrable, and if coordinate functions $y^{r_{i-1}+1}, \cdots, y^{r_{i}}$ associated to the integrable $G_{i}$-structure around the point $\left(x^{r_{i-1}+1}, \cdots, x^{r_{i}}\right)=\left(\xi^{r_{i-1}+1}, \cdots, \xi^{r_{i}}\right)$ are dependent on coordinates $x^{r_{i-1}+1}, \cdots, x^{r_{i}}$ and on parameters $x^{1}, \cdots, x^{r_{i-1}}, x^{r_{i}+1}, \cdots, x^{m}$ jointly in a $C^{\infty}$-manner, then we can replace $\left\{x^{1}, \cdots, x^{m}\right\}$ in a neighbourhood of the point $\left(x^{1}, \cdots, x^{m}\right)=\left(\xi^{1}, \cdots, \xi^{m}\right)$ by a new coordinate system $\left\{y^{1}, \cdots, y^{m}\right\}$, so that $h$ takes the matrix form $\mu$, i.e. $H$ is integrable.

Hence we consider the case where $h$ has characteristic polynomial $\{p(\lambda)\}^{a}$ and minimum polynomial $\{p(\lambda)\}^{v}$, where $p(\lambda)$ is irreducible over the reals, and suppose that $h$ jointly depends on the coordinates of $M$ and some parameters in a $C^{\infty}$-manner. We have the following results:

Case I. $\operatorname{deg} p(\lambda)=1$.

(i) If $v=1$, then $h$ is a constant multiple of the identity vector 1 -form $I$ on $M$, hence the $G$-structure is integrable.

(ii) If $v=d=m$, consider the nilpotent part $n$ of $h$. $n$ is a polynomial in $h$ with constant coefficients on $M$, so from $[h, h]=0$, we get $[n, n]=0$, by Lemma 1.1. Moreover $n^{m}=0$ but $n^{l} \neq 0$ for $l<m$, for all points of $M$. In $\S 3$ we prove a proposition which shows that the $G$-structure defined by $n$ (which is the same as that defined by $h$ ) is integrable, and that the associated coordinate functions depend on the parameters of $h$ and on the point in $M$ jointly in a $C^{\infty}$-manner.

Case II. $\operatorname{deg} p(\lambda)=2$. In $\S 4$ we shall show that the semi-simple part $s$ of $h$ gives rise to a complex manifold structure $\widetilde{M}$ in this case, and that for the $\widetilde{G}$-structure given by $h$ which is induced from $h$ on $\widetilde{M}$, (i) and (ii) of Case I has a straightforward parallel on $\widetilde{M}$; hence coming back to the real manifold, we have: if $v=1$, or $v=d=m / 2$, then the $G$-structure defined by $h$ is integrable, and the associated coordinate functions are $C^{\infty}$ with respect to the coordinates on $M$ and the parameters jointly.

By the preceding arguments and the results in $\S 3$ and 4 , we can conclude the following:

THEOREM. Let $h$ be a 0-deformable vector 1-form on a manifold $M$, with characteristic polynomial

$$
\prod_{i=1}^{g} p_{i}(\lambda)^{a_{i}}
$$

where $p_{i}(\lambda)$ are polynomials in $\lambda$, irreducible over the reals, and $\left(p_{i}(\lambda)\right.$, $\left.p_{j}(\lambda)\right)=1$ for $i \neq j$, and the minimum polynomial

$$
\prod_{i=1}^{g} p_{i}(\lambda)^{v_{i}}
$$


Suppose for each $i, v_{i}=1$ or $d_{i}$. Then the G-structure defined by $h$ is integrable if $[h, h]=0$.

REMARK. If $v_{i}=1$ for all $i$, we say that $h$ is semi-simple. If $v_{i}=d_{i}$ for all $i$, we say that $h$ is nonderogatory, and otherwise derogatory [6, p. 21].

\section{The integrability of a nonderogatory nilpotent vector 1-form.}

Proposition. Let h be a nilpotent vector 1 -form on an m-dimensional manifold $M$, and suppose $h^{m}=0$ but $h^{l} \neq 0$ for $l<m$, for all points on $M$. Then $[h, h]=0$ implies that the G-structure defined by $h$ is integrable. Moreover, if $h$ depends on some parameters and is $C^{\infty}$ with respect to the local coordinates $x^{1}, \cdots, x^{m}$ on $M$ and the parameters jointly, then the local coordinates $y^{1}, \cdots, y^{m}$ associated to the integrable G-structure are $C^{\infty}$ with respect to $x^{1}, \cdots, x^{m}$ and the parameters jointly.

Proof. (1) Let $m=2$. Denoting the tangent space at $x \in M$ by $T_{x}$, we have a one dimensional distribution given by $x \rightarrow h_{x} T_{x}$. For each point $x_{0}$ of $M$ we can find a neighbourhood $U$ of $x_{0}$ and a coordinate system $\left\{x^{1}, x^{2}\right\}$ on $U$, such that $x^{2}=\xi^{2}$ is an integral manifold of this distribution in $U$. Let $h$ take the matrix form in this coordinate system

$$
\left(\begin{array}{ll}
\beta_{11} & \beta_{12} \\
\beta_{21} & \beta_{22}
\end{array}\right)
$$

$\beta_{i j}$ being functions of $x^{1}, x^{2}$. As $\partial / \partial x^{1}$ at $x \in U$ spans $h_{x} T_{x}$, we have $\beta_{21}=\beta_{22}=0$, and as $h$ restricted to integral manifold $x^{2}=\xi^{2}$ is given by $\beta_{11}$, and as $h^{2}=0$, we have $\beta_{11}=0$. We claim, that we can choose a new coordinate system $\left\{y^{1}, y^{2}\right\}$ such that in this new coordinate system $h$ takes the matrix form

$$
\left(\begin{array}{ll}
0 & 1 \\
0 & 0
\end{array}\right)
$$

In fact, let the vector fields $\partial / \partial x^{1}$ and $\partial / \partial x^{2}$ be denoted by $X_{1}$ and $X_{2}$, and choose new vector fields $Y_{1}$ and $Y_{2}$ by

$$
\left\{\begin{array}{l}
Y_{1}=\alpha_{1} X_{1} \\
Y_{2}=\alpha_{0} X_{1}+X_{2}
\end{array}\right.
$$

where $\alpha_{1}$ and $\alpha_{0}$ are to be determined so that $h Y_{2}=Y_{1}$ and $\left[Y_{1}, Y_{2}\right]=0$. Let then $\pi^{1}, \pi^{2}$ be the 1 -forms dual to $Y_{1}, Y_{2}$; we have $d \pi^{1}=0, d \pi^{2}=0$, so that $y^{1}, y^{2}$ can be determined from $d y^{1}=\pi^{1}, d y^{2}=\pi^{2}$. To prove that $Y_{1}$ and $Y_{2}$ can be found we observe that the condition $h Y_{2}=Y_{1}$ leads to 


$$
\alpha_{1}=\beta_{12}
$$

and that the condition $\left[Y_{1}, Y_{2}\right]=0$ leads to

$$
\left(\alpha_{0} X_{1}+X_{2}\right) \alpha_{1}-\alpha_{1} X_{1} \alpha_{0}=0
$$

which is a first order linear differential equation for $\alpha_{0}$ :

$$
\alpha_{1} \frac{\partial}{\partial x^{1}} \alpha_{0}-\alpha_{0}\left(\frac{\partial}{\partial x^{1}} \alpha_{1}\right)-\frac{\partial}{\partial x^{2}} \alpha_{1}=0 \text {. }
$$

$\alpha_{1}$ is clearly $C^{\infty}$ with respect to $x^{1}, x^{2}$ and the parameters. $\alpha_{0}$ is obtained as a solution of the above differential equation, so $\alpha_{0}$ depends on $x^{2}$ and the parameters in a $C^{\infty}$ manner. By differentiating this differential equation repeatedly, we see that $\alpha_{0}$ is $C^{\infty}$ with respect to $x^{1}, x^{2}$ and the parameters. Hence $\pi^{1}$ and $\pi^{2}$ are $C^{\infty}$ with respect to $x^{1}, x^{2}$ and the parameters, and finally $y^{1}$ and $y^{2}$ are $C^{\infty}$ with respect to $x^{1}, x^{2}$ and the parameters.

(2) We assume that our proposition is true for $(m-1)$-dimensional manifolds and proceed to prove it for an $m$-dimensional manifold ( $m \geqq 3$ ).

Because $[h, h]=0$, we know that the distribution $x \rightarrow h_{x} T_{x}$, given by the image of $h$ at each point $x$ of $M$ is integrable; hence, locally, there exists a coordinate system $\left\{x^{1}, \cdots, x^{m}\right\}$ such that

(i) $x^{m}=\xi^{m}$ gives the integral manifolds of this distribution, and

(ii) in this coordinate system $h$ takes the matrix form

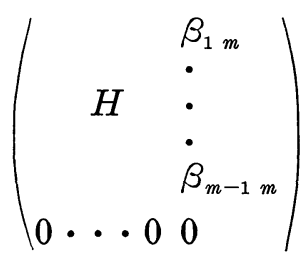

We further claim that $x^{1}, \cdots, x^{m-1}, x^{m}$ can be chosen so that

(iii) $H$ takes the form

$$
\left(\begin{array}{ccccc}
0 & 1 & 0 & \cdot & 0 \\
\cdot & 0 & 1 & \cdot & 0 \\
\cdot & & \cdot & \cdot & \cdot \\
\cdot & & & 0 & 1 \\
0 & \cdot & \cdot & \cdot & 0
\end{array}\right)
$$

In fact, if $H$ is not in the form (2) already, we view the restriction $h_{1}$ of $h$ to an integral manifold $x^{m}=\xi^{m}$ as a vector 1-form on an open set $V$ of $R^{m-1}$, depending on parameter $x^{m}$, and consider $H$ to be the matrix form of $h_{1}$ with respect to the coordinate system $\left\{x^{1}, \cdots, x^{m-1}\right\}$. From the inductive assumption, there are coordinate functions $z^{1}, \cdots, z^{m-1}$ on an open set $V_{1} \subset V$ depending on $x^{1}, \cdots, x^{m-1}$ and $x^{m}$ in a $C^{\infty}$-manner, such that $h_{1}$ has matrix form (2) with respect to the coordinate system 
$\left\{z^{1}, \cdots, z^{m-1}\right\}$. Now, if we take $\left\{z^{1}, \cdots, z^{m-1}, x^{m}\right\}$ as the local coordinate system on $M$, then (iii) will be satisfied.

So let us suppose that we are in a coordinate system where (i) (ii) and (iii) are satisfied. For simplicity we write $\beta_{1}, \beta_{2}, \cdots, \beta_{m-1}$ instead of $\beta_{1 m}, \beta_{2 m}, \cdots, \beta_{m-1 m}$. Note that $\beta_{m-1} \neq 0$. We want to prove that we can find a new coordinate system $\left\{y^{1}, \cdots, y^{m}\right\}$ such that in this coordinate system $h$ takes the matrix form (1), $H$ being of the form (2) and $\beta_{1}=\beta_{2}=\cdots=\beta_{m-2}=0, \beta_{m-1}=1$. In order to do this, as in the case $m=2$, we find vector fields $Y_{1}, \cdots, Y_{m}$ satisfying $h Y_{i}=Y_{i-1}(i=2, \cdots, m)$, $h Y_{1}=0$ and $\left[Y_{i}, Y_{j}\right]=0$ for all $i, j$; let the dual of $Y_{1}, \cdots, Y_{m}$ be $\pi^{1}, \cdots, \pi^{m}$ and obtain $y^{1}, \cdots, y^{m}$ from $d y^{1}=\pi^{1}, \cdots, d y^{m}=\pi^{m}$. If we denote by $X_{1}, \cdots, X_{m}$ the vector fields $\partial / \partial x^{1}, \cdots, \partial / \partial x^{m}$ and set

$$
\left\{\begin{array}{l}
Y_{1}=\alpha_{m-1} X_{1} \\
Y_{2}=\alpha_{m-2} X_{1}+\alpha_{m-1} X_{2} \\
\quad \cdots \cdots \cdots \\
Y_{m-1}=\alpha_{1} X_{1}+\alpha_{2} X_{2}+\cdots+\alpha_{m-1} X_{m-1} \\
Y_{m}=\alpha_{0} X_{1}+\left(\alpha_{1}-\beta_{1}\right) X_{2}+\cdots+\left(\alpha_{m-2}-\beta_{m-2}\right) X_{m-1}+X_{m}
\end{array}\right.
$$

where $\alpha_{m-1}=\beta_{m-1}$, then the problem reduces to finding the $\alpha$ 's so that $\left[Y_{i}, Y_{j}\right]=0$ are satisfied for all $i, j$.

First we shall obtain all the relations on the derivatives of $\beta_{1}, \cdots$, $\beta_{m-1}$ imposed by the condition $[h, h]=0$. We see that

$$
[h, h]\left(X_{i}, X_{j}\right)=0
$$

gives us no relations for $i, j \leqq m-1$, but

$$
\begin{aligned}
\frac{1}{2}[h, h]\left(X_{i}, X_{m}\right) & =\left[X_{i-1}, \beta_{1} X_{1}+\cdots+\beta_{m-1} X_{m-1}\right] \\
& -h\left[X_{i}, \beta_{1} X_{1}+\cdots+\beta_{m-1} X_{m-1}\right]
\end{aligned}
$$

from which we obtain

$$
X_{i-1} \beta_{j-1}=X_{i} \beta_{j} \quad i, j \leqq m-1
$$

and

$$
X_{i} \beta_{m-1}=0
$$$$
i \leqq m-2
$$

To make this relation clear, we write this result in Table 1. 


$$
\begin{aligned}
& 0=X_{1} \beta_{m-1} \\
& 0=X_{1} \beta_{m-2}=X_{2} \beta_{m-1} \\
& 0=X_{1} \beta_{3}=X_{2} \beta_{4}=\cdots \cdots \cdots \cdots X_{m-3} \beta_{m-1} \\
& 0=X_{1} \beta_{2}=X_{2} \beta_{3}=\ldots \ldots \ldots \ldots \ldots X_{m-3} \beta_{m-2}=X_{m-2} \beta_{m-1} \\
& X_{1} \beta_{1}=X_{2} \beta_{2}=\cdots \cdots \cdots \cdots X_{m-3} \beta_{m-3}=X_{m-2} \beta_{m-2}=X_{m-1} \beta_{m-1} \\
& X_{2} \beta_{1}=\ldots \ldots \ldots \ldots X_{m-3} \beta_{m-4}=X_{m-2} \beta_{m-3}=X_{m-1} \beta_{m-2} \\
& X_{m-3} \beta_{1}=X_{m-2} \beta_{2}=X_{m-1} \beta_{3} \\
& X_{m-2} \beta_{1}=X_{m-1} \beta_{2}
\end{aligned}
$$

TABLE 1

Now let us examine $\left[Y_{i}, Y_{j}\right]=0$ for $i<j \leqq m-1$. We see that this is equivalent to the set of equations (6),

(6)

$$
\left\{\begin{array}{c}
\left(\alpha_{m-i} X_{1}+\alpha_{m-i+1} X_{2}+\cdots+\alpha_{m-1} X_{i}\right) \alpha_{m-1}=0 \\
\ldots \ldots \\
\left(\alpha_{m-i} X_{1}+\alpha_{m-i+1} X_{2}+\cdots+\alpha_{m-1} X_{i}\right) \alpha_{m-j+i}=0 \\
\left(\alpha_{m-i} X_{1}+\alpha_{m-i+1} X_{2}+\cdots+\alpha_{m-1} X_{i}\right) \alpha_{m-j+i-1} \\
-\left(\alpha_{m-j} X_{1}+\alpha_{m-j+1} X_{2}+\cdots+\alpha_{m-1} X_{j}\right) \alpha_{m-1}=0 \\
\cdots \\
\left.\quad \cdots \cdots+\alpha_{m-1} X_{i}\right) \alpha_{m-j} \\
-\left(\alpha_{m-j} X_{1}+\alpha_{m-j+1} X_{2}+\cdots+\alpha_{m-1} X_{j}\right) \alpha_{m-i}=0
\end{array}\right.
$$

where $i<j \leqq m-1$. Using $X_{1} \alpha_{m-1}=X_{1} \beta_{m-1}=0$ from Table 1 , we see that (6) is equivalent to the following Table 2.

$$
\left.\begin{array}{r}
0=X_{1} \alpha_{m-1} \\
0=X_{1} \alpha_{m-2}=X_{2} \alpha_{m-1} \\
\cdots \cdots \\
\begin{array}{rl}
0=X_{1} \alpha_{3}=X_{2} \alpha_{4}=\cdots \\
0=X_{1} \alpha_{2}=X_{m-3} \alpha_{m-1}
\end{array} \\
X_{1} \alpha_{1}=\cdots=X_{m-3} \alpha_{m-2}=X_{m-2} \alpha_{m-1} \\
X_{2} \alpha_{2}=\cdots=X_{m-4} \alpha_{m-3}=X_{m-2} \alpha_{m-2}=X_{m-1} \alpha_{m-1} \\
X_{2} \alpha_{1}=\cdots=X_{m-3} \alpha_{m-4}=X_{m-2} \alpha_{m-3}=X_{m-1} \alpha_{m-2} \\
\ldots \ldots \ldots \\
X_{m-3} \alpha_{1}=X_{m-2} \alpha_{2}=X_{m-1} \alpha_{3} \\
X_{m-2} \alpha_{1}=X_{m-1} \alpha_{2}
\end{array}\right\}
$$

TABLE 2

Next consider $\left[Y_{i}, Y_{m}\right]=0, i \leqq m-1$. This is equivalent to the following $(7 a, b, c)$, 
(7a)

$$
\begin{gathered}
\left\{\begin{array}{c}
\left(\alpha_{m-i} X_{1}+\alpha_{m-i+1} X_{2}+\cdots+\alpha_{m-1} X_{i}\right)\left(\alpha_{m-2}-\beta_{m-2}\right)=0 \\
\cdots \cdots \\
\left(\alpha_{m-i} X_{1}+\alpha_{m-i+1} X_{2}+\cdots+\alpha_{m-1} X_{i}\right)\left(\alpha_{i}-\beta_{i}\right)=0
\end{array}\right. \\
\left\{\begin{array}{c}
\left(\alpha_{m-i} X_{1}+\alpha_{m-i+1} X_{2}+\cdots+\alpha_{m-1} X_{i}\right)\left(\alpha_{i-1}-\beta_{i-1}\right) \\
-\left\{\alpha_{0} X_{1}+\left(\alpha_{1}-\beta_{1}\right) X_{2}+\cdots+\left(\alpha_{m-2}-\beta_{m-2}\right) X_{m-1}+X_{m}\right\} \alpha_{m-1}=0 \\
\cdots \cdots \cdots
\end{array}\right. \\
\left(\begin{array}{c} 
\\
\left(\alpha_{m-i} X_{1}+\alpha_{m-i+1} X_{2}+\cdots+\alpha_{m-1} X_{i}\right)\left(\alpha_{1}-\beta_{1}\right) \\
-\left\{\alpha_{0} X_{1}+\left(\alpha_{1}-\beta_{1}\right) X_{2}+\cdots+\left(\alpha_{m-2}-\beta_{m-2}\right) X_{m-1}+X_{m}\right\} \alpha_{m-i+1}=0
\end{array}\right. \\
\left(\alpha_{m-i} X_{1}+\alpha_{m-i+1} X_{2}+\cdots+\alpha_{m-1} X_{i}\right) \alpha_{0} \\
-\left\{\alpha_{0} X_{1}+\left(\alpha_{1}-\beta_{1}\right) X_{2}+\cdots+\left(\alpha_{m-2}-\beta_{m-2}\right) X_{m-1}+X_{m}\right\} \alpha_{m-i}=0
\end{gathered}
$$

(7b)

where $i \leqq m-1$.

Because of Table 1, we see that (7a) is equivalent to part (a) of Table 2. Using part (a) of Table 2 , we see that (7b) reduces to a simpler system $\left(7 b^{\prime}\right)$,

$\left(7 b^{\prime}\right)$

$$
\begin{aligned}
& \left(\begin{array}{l}
\left(\alpha_{m-1} X_{i}\right)\left(\alpha_{i-1}-\beta_{i-1}\right)-\left\{\left(\alpha_{m-2}-\beta_{m-2}\right) X_{m-1}+X_{m}\right\} \alpha_{m-1}=0 \\
\left(\alpha_{m-2} X_{i-1}+\alpha_{m-1} X_{i}\right)\left(\alpha_{i-2}-\beta_{i-2}\right)-\left\{\left(\alpha_{m-3}-\beta_{m-3}\right) X_{m-2}\right.
\end{array}\right. \\
& \left.+\left(\alpha_{m-2}-\beta_{m-2}\right) X_{m-1}+X_{m}\right\} \alpha_{m-2}=0 \\
& \left(\alpha_{m-i+1} X_{2}+\cdots+\alpha_{m-1} X_{i}\right)\left(\alpha_{1}-\beta_{1}\right) \\
& -\left\{\left(\alpha_{m-i}-\beta_{m-i}\right) X_{m-i+1}+\cdots+\left(\alpha_{m-2}-\beta_{m-2}\right) X_{m-1}+X_{m}\right\} \alpha_{m-i+1}=0
\end{aligned}
$$

Using Table 1 again, we can show that $\left(7 b^{\prime}\right)$ is equivalent to part (b) of Table 2 plus the following equations which are obtained from $\left(7 b^{\prime}\right)$ by letting $i=m-1$ :

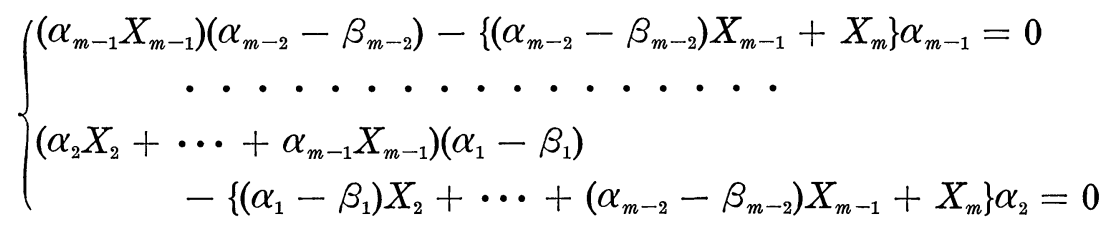

Using Table 1 and part (b) of Table 2, these equations can be written as (8),

$$
\left(\alpha_{m-1}\right)^{2} X_{m-1} \frac{\alpha_{m-k}-\beta_{m-k}}{\alpha_{m-1}}+\left(\alpha_{m-2}\right)^{2} X_{m-1} \frac{\alpha_{m-k+1}-\beta_{m-k+1}}{\alpha_{m-2}}
$$

$+\cdots+\left(\alpha_{m-k+1}\right)^{2} X_{m-1} \frac{\alpha_{m-2}-\beta_{m-2}}{\alpha_{m-k+1}}-X_{m} \alpha_{m-k+1}=0,{ }^{1)} \quad k=2, \cdots, m-1$.

1 For simplicity we write $\left(\alpha_{m-1-j}\right)^{2} X_{m-1}\left(\alpha_{m-k+j}-\beta_{m-k+j} / \alpha_{m-1-j}\right), 1 \leqq j \leqq k-2$, for $\alpha_{m-1-j} X_{m-1}\left(\alpha_{m-k+j}-\beta_{m-k+j}\right)-\left(X_{m-1} \alpha_{m-1-j}\right) X_{m-1}\left(\alpha_{m-k+j}-\beta_{m-k+j}\right)$, although at some point $\alpha_{m-1-j}$ might vanish. 
We can now obtain $\alpha_{m-2}, \alpha_{m-3}, \cdots, \alpha_{1}$ succesively by integrating (8) with respect to $x^{m-1}$; in fact, start from $k=2$, and integrate to get $\alpha_{m-2}$, then use this $\alpha_{m-2}$ in (8) for $k=3$ and integrate to get $\alpha_{m-3}$, in general

$$
\begin{aligned}
& \alpha_{m-k}-\beta_{m-k}=\alpha_{m-1} \int \frac{-1}{\left(\alpha_{m-1}\right)^{2}}\left\{\left(\alpha_{m-2}\right)^{2} X_{m-1} \frac{\alpha_{m-k+1}-\beta_{m-k+1}}{\alpha_{m-2}}\right. \\
&\left.+\cdots+\left(\alpha_{m-k+1}\right)^{2} X_{m-1} \frac{\alpha_{m-2}-\beta_{m-2}}{\alpha_{m-k+1}}-X_{m} \alpha_{m-k+1}\right\} d x^{m-1} .
\end{aligned}
$$

We still have to show that $\alpha_{m-2}, \alpha_{m-3}, \cdots, \alpha_{1}$ thus obtained satisfy Table 2. For simplicity let us write (8) in the form

$$
\left(\alpha_{m-1}\right)^{2} X_{m-1} \frac{\alpha_{m-k}-\beta_{m-k}}{\alpha_{m-1}}+A_{m-k+1}=0 .
$$

Then (9) becomes

$$
\alpha_{m-k}-\beta_{m-k}=\alpha_{m-1} \int \frac{-1}{\left(\alpha_{m-1}\right)^{2}} A_{m-k+1} d x^{m-1} .
$$

To show that the $\alpha$ 's do satisfy Table 2 , it suffices to show $\left(10_{k}\right)$,

$$
X_{m-q}\left(\alpha_{m-k}-\beta_{m-k}\right)=X_{m-q+1}\left(\alpha_{m-k+1}-\beta_{m-k+1}\right)
$$

for $k, q=2, \cdots, m-1$. We shall prove $\left(10_{k}\right)$ inductively. For $k=2$ it is easy to check. Suppose $\left(10_{2}\right), \cdots,\left(10_{k-1}\right)$ are true; using this assumption, we differentiate $\left(9_{k}\right)$ and get (11),

$$
\begin{gathered}
X_{m-q}\left(\alpha_{m-k}-\beta_{m-}\right)=a_{m-1} \int \frac{-1}{\left(\alpha_{m-1}\right)^{2}}\left\{\left(X_{m-q m-2} \alpha\right)^{2} X_{m-1} \frac{\alpha_{m-k+1}-\beta_{m-k+1}}{X_{m-q} \alpha_{m-2}}\right. \\
\left.+X_{m-q+1} A_{m-k+2}+\left(\alpha_{m-k+1}\right)^{2} X_{m-1} \frac{X_{m-q}\left(\alpha_{m-2}-\beta_{m-2}\right)}{\alpha_{m-k+1}}\right\} d x^{m-1}
\end{gathered}
$$

If $q>2$, then $X_{m-q} \alpha_{m-2}=0$, so (11) gives us $\left(10_{k}\right)$. If $q=2$, we observe first that differentiating $\left(8_{k+1}\right)$ with respect to $x^{m-1}$ gives us (12),

$$
\begin{gathered}
\left(X_{m-1}^{2}\left(\alpha_{m-k+1}-\beta_{m-k+1}\right)\right) \alpha_{m-1}-\left(\alpha_{m-k+1}-\beta_{m-k+1}\right) X_{m-1}^{2} \alpha_{m-1} \\
+X_{m-1} A_{m-k+2}=0 .
\end{gathered}
$$

Using (12) and $X_{m-2}\left(\alpha_{m-2}-\beta_{m-2}\right)=0$ in (11) for $q=2$, we obtain

$$
\begin{gathered}
X_{m-2}\left(\alpha_{m-k}-\beta_{m-k}\right)=\alpha_{m-1} \int \frac{-1}{\left(\alpha_{m-1}\right)^{2}}\left\{\left(X_{m-1}\left(\alpha_{m-k+1}-\beta_{m-k+1}\right)\right) X_{m-1} \alpha_{m-1}\right. \\
\left.-\left(X_{m-1}^{2}\left(\alpha_{m-k+1}-\beta_{m-k+1}\right)\right) \alpha_{m-1}\right\} d x^{m-1}=X_{m-1}\left(\alpha_{m-k+1}-\beta_{m-k+1}\right)
\end{gathered}
$$

which completes the proof $\left(10_{k}\right)$.

Finally to obtain $\alpha_{0}$, we examine (7c), and find that the same type of argument employed to obtain (8) enables us to show that (7c) is equivalent to 


$$
\left\{\begin{array}{l}
X_{1} \alpha_{0}=X_{m-1}\left(\alpha_{m-2}-\beta_{m-2}\right) \\
\cdot \cdot \cdot \cdot \cdot \\
X_{m-2} \alpha_{0}=X_{m-1}\left(\alpha_{1}-\beta_{1}\right) \\
\left(\alpha_{1} X_{1}+\cdots+\alpha_{m-1} X_{m-1}\right) \alpha_{0}-\left\{\alpha_{0} X_{1}+\left(\alpha_{1}-\beta_{1}\right) X_{2}+\right. \\
\left.\cdots+\left(\alpha_{m-2}-\beta_{m-2}\right) X_{m-1}+X_{m}\right\} \alpha_{m-1}=0 .
\end{array}\right.
$$

Using the first $m-2$ equations of (13) in the last one, gives us $\left(8_{k}\right)$ for $k=m$, where we agree that $\beta_{0}=0$. Hence we obtain $\alpha_{0}$ from $\left(9_{m}\right)$. To check that the first $m-2$ equations in (13) are satisfied by this $\alpha_{0}$, we check $\left(10_{k}\right)$ for $k=m$. The same argument in (11) holds for $k=m$, and it is even simpler than before, because in this case the first term in the integrand vanishes.

If $h$ depends on $x^{1}, \cdots, x^{m}$ and some parameters jointly in a $C^{\infty_{-}}$ manner, then it is clear that $\alpha_{m-2}, \cdots, \alpha_{1}, \alpha_{0}$ obtained above depend on $x^{1}, \cdots, x^{m}$ and the parameters in a $C^{\infty}$-manner, hence we can claim the same for $y^{1}, \cdots, y^{m}$.

4. The complex case. For Case II in $\S 2$, where $\operatorname{deg} p(\lambda)=2$, we have $\operatorname{dim} M=m=2 n$. Let the roots of $p(\lambda)=0$ be $\sigma \pm i \tau(\tau \neq 0)$. Because the semi-simple part $s$ of $h$ is a polynomial in $h$ with constant coefficients, from $[h, h]=0$, via Lemma 1.1 , we get $[s, s]=0$. The vector 1-form $J_{s}$ defined by

$$
J_{s}=\frac{1}{\tau}(s-\sigma I)
$$

satisfies $\lambda^{2}+1=0$, because $s$ satisfies $p(\lambda)=0$. So we have an almost complex structure $J_{s}$ on $M$, and as $\left[J_{s}, J_{s}\right]=0$ (because $[s, s]=0$ ), this almost complex structure is integrable [7]. Hence we can introduce a new real local coordinate system $\left\{x^{1}, \cdots, x^{m}\right\}$ such that $z^{k}=x^{2 k-1}+i x^{2 k}$ $(k=1, \cdots, n)$ gives a local complex coordinate system, with which $M$ becomes the underlying $C^{\infty}$-manifold of complex manifold $\widetilde{M}$. As $h$ is $C^{\infty}$ with respect to the coordinates on $M$ and the parameters jointly, so is the almost complex structure $J_{s}$. Hence the new coordinate functions $x^{1}, \cdots, x^{m}$ are also $C^{\infty}$ with respect to the coordinates on $M$ and the parameters jointly [7]. ${ }^{2} h$ is now $C^{\infty}$ with respect to $x^{1}, \cdots, x^{m}$ and the parameters jointly. The vector 1 -forms on $M$ induce vector 1 -forms on $\widetilde{M}$ in a natural way. The vector 1 -form $\widetilde{s}$ on $\widetilde{M}$ induced by $s$ is equal to $\rho \widetilde{I}$, where $\rho=\sigma+i \tau$ and $\widetilde{I}$ is the identity vector 1 -from on $\widetilde{M}$. We shall show that polynomials in $h$ with constant coefficients induce holomorphic vector 1-forms on $M$. In particular, the nilpotent part $n$ of $h$ induces the nilpotent holomorphic vector 1 -form $\tilde{n}$ on $M$.

2 The author wishes to thank Professor L. Nirenberg for communicating the proof of this fact to him. The dependence on parameters is stated without proof in [7]. 
Let $T_{\sigma}$ and $T_{o}^{(p)}$ be the vector bundles over $M$, which are obtained by complexifying the tangent space $T_{x}$ and the space of tangential covariant $p$-vectors $T_{x}^{(p)}$ respectively at each point $x$ of $M$. Then any $p$-form $P$ on $M$, i.e. any cross-section of $T \otimes T^{(p)}$, extends in a natural way to a cross-section $P_{o}$ of $T_{o} \otimes T_{o}^{(p)}$. If $k$ and $l$ are two vector 1forms on $M$, then $k_{\sigma}, l_{\sigma}$ and $[k, l]_{o}$ are defined. If we define the bracket of two cross-sections of $T_{o}$ in a natural way, and if we define $\left[k_{o}, l_{\sigma}\right]$ by (2) of $\S 1$, where we replace $h, k$ by $k_{\sigma}, l_{\sigma}$ acd $u, v$ by cross-sections of $T_{o}$, then we have $[k, l]_{o}=\left[k_{\sigma}, l_{\sigma}\right]$.

Denote $\partial / \partial \bar{z}^{i}, \partial / \partial z^{i}$ by $Z_{i}, \bar{Z}_{i}$ for $i=1, \cdots, n$. $\left(Z_{1}\right)_{x}, \cdots,\left(Z_{n}\right)_{x},\left(\bar{Z}_{1}\right)_{x}$, $\cdots,\left(\bar{Z}_{n}\right)_{x}$ span the complexification of $T_{x} .\left(Z_{1}\right)_{x}, \cdots,\left(Z_{n}\right)_{x}$ span the eigenspaces of eigenvalue $\rho$. This eigenspace can be identified with the tangent space of $\widetilde{M}$ at $x .\left(\bar{Z}_{1}\right)_{x}, \cdots,\left(\bar{Z}_{n}\right)_{x}$ span the eigenspace of $\left(s_{0}\right)_{x}$ of eigenvalue $\bar{\rho}$. If $k$ is a polynomial in $h$ with constant coefficients, by Lemma 1.1 we have $[s, k]=0$, and hence $\left[s_{\sigma}, k_{\sigma}\right]=[s, k]_{o}=0$. On the other hand we have

$$
\left[s_{o}, k_{\sigma}\right]\left(Z_{i}, \bar{Z}_{j}\right)=(\rho-s)\left[Z_{i}, k_{\sigma} \bar{Z}_{j}\right]+(\bar{\rho}-s)\left[k_{o} Z_{i}, \bar{Z}_{j}\right] .
$$

$s_{\sigma}$ and $k_{\sigma}$ are polynomials in $h_{\sigma}$ with constant coefficients, so $s_{\sigma}$ and $k_{\sigma}$ commute; hence $k_{\sigma}$ leave the eigenspaces of $s_{\sigma}$ invariant, so using the coordinate expression for $k_{o}$, the equation above can be written as

$$
\left[s_{o}, k_{\sigma}\right]\left(Z_{i}, \bar{Z}_{j}\right)=(\rho-\bar{\rho}) \sum_{k=1}^{n}\left\{\left(Z_{i}\left(k_{\sigma}\right)_{\bar{k} \bar{j}}\right) \bar{Z}_{k}+\left(\bar{Z}_{j}\left(k_{\sigma}\right)_{k i}\right) Z_{k}\right\}
$$

from which we get

$$
\left(\partial / \partial \bar{z}^{\jmath}\right)\left(k_{\sigma}\right)_{k i}=0 \text {. }
$$

$\left(k_{\sigma}\right)_{k i}$ is the matrix form of $\tilde{k}$ on $\widetilde{M}$ (induced by $k$ ) with respect to the coordinate system $\left\{z^{1}, \cdots, z^{n}\right\}$, and (1) expresses the fact that $\widetilde{k}$ is holomorphic. ${ }^{3}$

(i) If $v=1$ in Case II of $\S 2$, then $\widetilde{h}$ induced by $h$ on $\widetilde{M}$, is equal to $\widetilde{s}=\rho \widetilde{I}$. So in the real coordinate system $\left\{x^{1}, \cdots, x^{m}\right\} h$ takes the matrix form

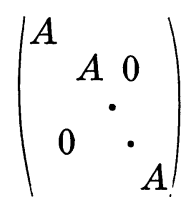

where

$$
A=\left(\begin{array}{lr}
\sigma & \tau \\
-\tau & \dot{\sigma}
\end{array}\right)
$$

${ }^{3}$ The author is indebted to Professor H. C. Wang for this proof. 
so that $G$-structure is integrable.

(ii) If $v=d=n$ in Case II of $\S 2$, then $\tilde{n}$ satisfies $\tilde{n}^{n}=0$ but $\widetilde{n}^{l} \neq 0$ for $l<n$ for all points on $\widetilde{M}$. As $\tilde{n}$ is holomorphic, it is meaningful to define the Nijenhuis tensor $[\tilde{n}, \tilde{n}]$ of $\tilde{n}$, using (3) of $\S 1$ as the defining formula, where $u, v$ should be holomorphic vector fields on $\tilde{M}$. As $\left[n_{o}, n_{o}\right]=[n, n]_{o}=0$, we have $[\tilde{n}, \tilde{n}]=0$.

Now following the method in $\S 3$, it is easy to see that we have a. complex version of the Proposition in $\S 3$, i.e.

"Let $\widetilde{k}$ be a holomorphic nilpotent vector 1-form on an $n$-dimensional complex manifold, and suppose $\widetilde{k}^{n}=0$ but $\tilde{k}^{l}=0$ for $l<n$, for all points. Then $[\widetilde{k}, \widetilde{k}]=0$ implies that the $\widetilde{G}$-structure defined by $\widetilde{k}$ is integrable. Moreover, if $\widetilde{k}$ depends on some complex [real] parameters and is holomorphic $\left[C^{\infty}\right]$ with respect to the local coordinates $z^{1}, \cdots, z^{n}$ [the real coordinates $x^{1}, \cdots, x^{m}$, where $\left.z^{k}=x^{2 k-1}+i x^{2 k}\right]$ and the parameters jointly, then the local coordinates $w^{1}, \cdots, w^{n}$ associated to the integrable $\widetilde{G}$ structure [the real coordinates $y^{1}, \cdots, y^{m}$ obtained from $w^{k}=y^{2 k-1}+i y^{2 k}$ ] are holomorphic $\left[C^{\infty}\right]$ with respect to $z^{1}, \cdots, z^{n}\left[x^{1}, \cdots, x^{m}\right]$ and the parameters jointly."

Using this complex version, for each point of $\tilde{M}$, we have a neighbourhood with a local complex coordinate system $w^{1}, \cdots, w^{n}$, with respect. to which $\widetilde{h}=\widetilde{s}+\widetilde{n}$ takes the matrix form

$$
\left(\begin{array}{lllll}
\rho & 1 & & & \\
& \rho & 1 & 0 \\
& & & : & \\
& 0 & & & \rho
\end{array}\right)
$$

Passing back to the real coordinate system $\left\{y^{1}, \cdots, y^{m}\right\}\left(w^{k}=y^{2 k-1}+i y^{2 k}\right)$, $h$ takes the matrix form

$$
\left|\begin{array}{cccc}
A & B & & \\
A & B & 0 \\
\cdot & \cdot & \\
& \bullet & \\
0 & & A & B \\
& & & A
\end{array}\right|
$$

where

$$
A=\left(\begin{array}{ll}
\sigma & \tau \\
-\tau & \sigma
\end{array}\right) \text { and } B=\left(\begin{array}{ll}
1 & 0 \\
0 & 1
\end{array}\right)
$$

The $G$-structure defined by $h$ is thus integrable. The associated local coordinates $y^{1}, \cdots, y^{m}$ are $C^{\infty}$-functions of the coordinates of $M$ and the parameters jointly.

5. An example. ${ }^{4}$ Let $M$ be the euclidean space of dimension 4 , and

${ }_{4}$ The author is indebted to Professor H. C. Wang for this example. 
suppose $x, y, z, t$ are the coordinates. Let

$$
X_{1}=\partial / \partial x, X_{2}=\partial / \partial y, X_{3}=\partial / \partial z, X_{4}=(\partial / \partial t)+(1+z)(\partial / \partial x),
$$

and define $h$ by $h X_{1}=X_{2}, h X_{i}=0$ for $i=2,3,4$. It is easy to check that

$$
\begin{aligned}
& \text { (i) } h^{2}=0, \\
& \text { (ii) }[h, h]=0,
\end{aligned}
$$

and (iii) $\left[X_{3}, X_{4}\right]=X_{1}$.

Now, if the $G$-structure defined by $h$ would be integrable, so would the distributions intrinsically given by $h$. However, (iii) shows that the distribution given by the kernel of $h$ at each point of $M$ is not integrable, hence we conclude that the $G$-structure is not integrable.

\section{REFERENCES}

1. D. Bernard, Sur la géometrie différentielles des G-structures, Ann. Inst. Fourier, Grenoble, 10 (1960), 153-273.

2. E. Cartan, Sur le problème général de la déformation, C. R. Congrès Strasbourg, (1920), 397-406 (Oeuvres Complètes III, vol. 1).

3. B. Eckmann et A. Frölicher, Sur l'intégrabilité des structures presque complexes, C. R. Paris, 232 (1951), 2284-2286.

4. A. Frölicher and A. Nijenhuis, Theory of vector-valued differential forms $I$, Proc. Kon. Ned. Ak. Wet. Amsterdam, A 59(3), (1956), 338-359.

5. N. Jacobson, Lectures in Abstract Algebra, vol. II, New York, 1953.

6. C. C. MacDuffee, The Theory of Matrices, Berlin, 1933.

7. A. Newlander and L. Nirenberg, Complex analytic coordinates in almost complex manifolds, Ann. of Math., (2) 65 (1957), 391-404.

NORTHWESTERN UNIVERSITY 



\title{
PACIFIC JOURNAL OF MATHEMATICS
}

\author{
EDITORS
}

RalPh S. Phillips

Stanford University

Stanford, California

M. G. Arsove

University of Washington

Seattle 5 , Washington
A. L. Whiteman

University of Southern California Los Angeles 7, California

LOWell J. PAIGE

Unıversity of California

Los Angeles 24, California

\section{ASSOCIATE EDITORS}
E. F. BECKENBACH
D. DERRY
H. L. ROYDEN
E. G. STRAUS
T. M. CHERRY
M. OHTSUKA
E. SPANIER
F. WOLF

\section{SUPPORTING INSTITUTIONS}

\author{
UNIVERSITY OF BRITISH COLUMBIA \\ CALIFORNIA INSTITUTE OF TECHNOLOGY \\ UNIVERSITY OF CALIFORNIA \\ MONTANA STATE UNIVERSITY \\ UNIVERSITY OF NEVADA \\ NEW MEXICO STATE UNIVERSITY \\ OREGON STATE UNIVERSITY \\ UNIVERSITY OF OREGON \\ OSAKA UNIVERSITY \\ UNIVERSITY OF SOUTHERN CALIFORNIA
}

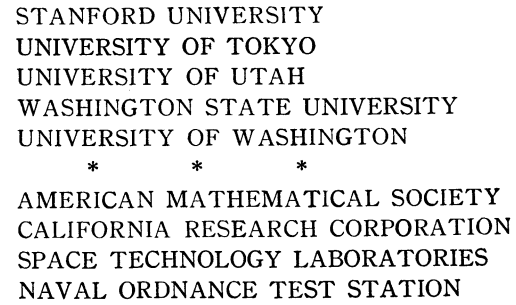

Mathematical papers intended for publication in the Pacific Journal of Mathematıcs should be typewritten (double spaced), and the author should keep a complete copy. Manuscripts may be sent to any one of the four editors. All other communications to the editors should be addressed to the managing editor, L. J. Paige at the University of California, Los Angeles 24, California.

50 reprints per author of each article are furnıshed free of charge; additional copies may be obtained at cost in multiples of 50 .

The Pacific Journal of Mathematics is published quarterly, in March, June, September, and December. Effective with Volume 13 the price per volume (4 numbers) is $\$ 18.00$; single issues, $\$ 5.00$. Special price for current issues to individual faculty members of supporting institutions and to individual members of the American Mathematical Society: $\$ 8.00$ per volume; single issues $\$ 2.50$. Back numbers are available.

Subscriptions, orders for back numbers, and changes of address should be sent to Pacific Journal of Mathematics, 103 Highland Boulevard, Berkeley 8, California.

Printed at Kokusai Bunken Insatsusha (International Academic Printing Co., Ltd.), No. 6 , 2-chome, Fujimi-cho, Chiyoda-ku, Tokyo, Japan.

PUBLISHED BY PACIFIC JOURNAL OF MATHEMATICS, A NON-PROFIT CORPORATION

The Supporting Institutions listed above contribute to the cost of publication of this Journal, but they are not owners or publishers and have no responsibility for its content or policies. 


\section{Pacific Journal of Mathematics}

\section{Vol. 12, No. $3 \quad$ March, 1962}

Alfred Aeppli, Some exact sequences in cohomology theory for Kähler

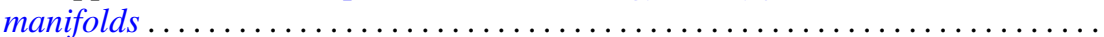

Paul Richard Beesack, On the Green's function of an N-point boundary value

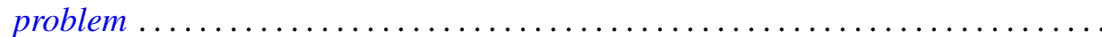

James Robert Boen, On p-automorphic p-groups....

James Robert Boen, Oscar S. Rothaus and John Griggs Thompson, Further results

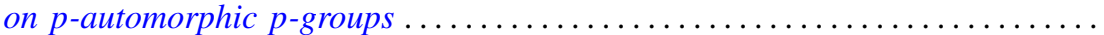

James Henry Bramble and Lawrence Edward Payne, Bounds in the Neumann problem for second order uniformly elliptic operators ..................

Chen Chung Chang and H. Jerome (Howard) Keisler, Applications of ultraproducts of pairs of cardinals to the theory of models .........................

Stephen Urban Chase, On direct sums and products of modules ................

Paul Civin, Annihilators in the second conjugate algebra of a group algebra .......

J. H. Curtiss, Polynomial interpolation in points equidistributed on the unit

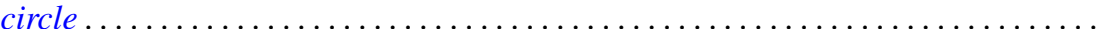

Marion K. Fort, Jr., Homogeneity of infinite products of manifolds with

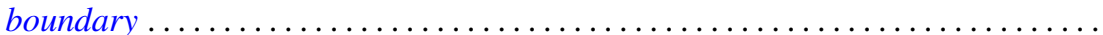

James G. Glimm, Families of induced representations . . . . . . . . . . . . . . .

Daniel E. Gorenstein, Reuben Sandler and William H. Mills, On almost-commuting

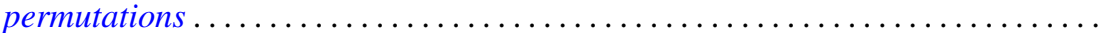

Vincent C. Harris and M. V. Subba Rao, Congruence properties of $\sigma_{r}(N) \ldots \ldots \ldots$

Harry Hochstadt, Fourier series with linearly dependent coefficients . . . . . . . . . . 925

Kenneth Myron Hoffman and John Wermer, A characterization of $C(X)$. .

Robert Weldon Hunt, The behavior of solutions of ordinary, self-adjoint differential equations of arbitrary even order...

Edward Takashi Kobayashi, A remark on the Nijenhuis tensor

David London, On the zeros of the solutions of $w^{\prime \prime}(z)+p(z) w(z)=0$

Gerald R. Mac Lane and Frank Beall Ryan, On the radial limits of Blaschke products...

T. M. MacRobert, Evaluation of an E-function when three of its upper parameters

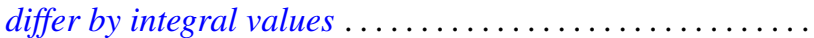

Robert W. McKelvey, The spectra of minimal self-adjoint extensions of a symmetric operator

Adegoke Olubummo, Operators of finite rank in a reflexive Banach space. .

David Alexander Pope, On the approximation of function spaces in the calculus of variations

Bernard W. Roos and Ward C. Sangren, Three spectral theorems for a pair of singular first-order differential equations...............

Arthur Argyle Sagle, Simple Malcev algebras over fields of characteristic zero .

S. C. Tang, Some theorems on the ratio of empirical distribution to the theoretical distribution

Robert Charles Thompson, Normal matrices and the normal basis in abelian number fields.

Howard Gregory Tucker, Absolute continuity of infinitely divisible distributions ... 